\title{
THE SER ANALYSIS OF RAYLEIGH, RICIAN AND NAKAGAMI CHANNELS AT VARIOUS RELAY LOCATIONS IN COOPERATIVE NETWORKS

\author{
SALMAN SALEEM ${ }^{1 *}$, HUMAIRA REHMAN $^{2}$, IMRAN KHAN $^{3}$, SAJJAD Ali $^{4}$, HASEEB AHMED K $_{\text {HAN }}{ }^{5}$ \\ ${ }^{1-5}$ Department of Telecommunication Engineering, University of Engineering and Technology \\ Peshawar, Pakistan \\ Email: *engrsalmansaleem@gmail.com
}

\begin{abstract}
Revised November 2015
ABSTRACT. Reliable communication between transmitter and receiver is accomplished by cooperative diversity techniques. Sending of data in various paths have greatly improved the performance of communication. We have studied the performance of Amplify-and-Forward (AF) based network in this work for diverse relay location at Nakagami, Rician and Rayleigh fading channels. The relay performance in Amplify-and-Forward (AF) protocol based on Symbol Error Rate (SER) against Signal-to-Noise Ratio (SNR) in dBs is calculated. The software that is used to construct Monte-Carlo link level simulation is MATLAB. The effects of relay at changed location in diverse channels accompanied with Additive White Gaussian noise (AWGN) is also calculated. BPSK modulation scheme is used for the transfer of information between the source, relay and destination node. The signals are combined through Maximum Ratio Combining method (MRC).

Keywords: Cooperative Communication; Amplify-and-Forward; Maximum Ratio Combining; Symbol Error Rate; AWGN; BPSK Modulation.
\end{abstract}

1. Introduction. Cooperative communication is the communication in which numerous users can share information to achieve spatial diversity with solitary antenna [1]. The information transmitting from source to destination have numerous fading effects. The diversity concept is used to transfer the data over multiple paths in order to lessen the fading effect during the transmission process. Space diversity (SD), Micro diversity (MiD), Frequency diversity (FD), Time diversity (TD) and Macro diversity (MaD) are some of the methods used to attain the diversity [2]. The most common and important protocol used in cooperative communication is Amplify-and-Forward (AF). The AF protocol amplifies the signal then passes (forwards) to the destination. Relay plays a significant part in maximizing the coverage area and have a dynamic role in long term Evolution (LTE) [3]. The asymptotic study of symbol error rate of Selective Amplify-and-Forward protocol (S-AF) is shown in [4]. It is verified that S-AF have full diversity gain in cooperative networks in terms of SER. The SER over Nakagami-m fading channel in cooperative communication for single relay is analyzed in [5]. In [6] the beam forming performance of two-way AF relay network is shown. The AF (variable-gain) communication accompanied channel estimation inaccuracies at the relay node and target (destination) terminal is examined in [7]. The tight estimates of the normal SER in the Rician fading channels are estimated for AF protocol in [8] of the opportunistic relay networks. Later on, tight estimations for the SER in average of duplication based networks for Nakagami fading channels in AF protocol are calculated in [9]. The AF protocol in common bidirectional relay networks with two sources and relays are shown in [10]. The AF protocol is mostly called $\mathrm{NC}$ at signal level [11, 12]. In [13], the comparison between various protocols in network-coded environment is studied. The relays are selected at various locations in [13]. The most important factor, which is considered in wireless networks, is fading which is classified into time variant and time spreading viewpoint. The Rayleigh Fading Channel, Rician Fading Channel, Nakagami Fading Channel and Additive White Gaussian Noise AWGN channels are mostly under consideration. The AWGN channels and Rician channel provides best performance in rural areas as compared to urban areas. Whereas, the Rayleigh channel provides premium efficiency in urban locality and poorer in rural areas. The Nakagami 
channel has greater elasticity and accuracy in experimental data in contrast with other channels.

In this paper, we have considered AF protocol over Rayleigh, Rician, and Nakagami fading channels. Additionally, the distance effect of relay over stated channels from the communicating nodes on the network at diverse location is studied. The SER results for various channels based on location of relay from the source are compared with each other. It is found that the performance of relay network changes with respect to the change in the Fading channel and locality of relays on the basis of Symbol Error Rate (SER).

The remaining of our work is organized as; the schematic diagram for the planned system is given in Section 2. Section 3 contains the simulation outcomes of the suggested network. Section 4 has some discussion on the outcomes. Lastly, in Section 5 we offer the conclusions along with the possible future works.

2. System Model. Our work consists of a wireless network publicized in figure 1, which contain a distinct source $(\boldsymbol{S})$ single relay $(\boldsymbol{R})$ and a destination $(\boldsymbol{D})$. There is a single antenna at each node. We use MRC to combine various signals at destination point. The information is transferred through orthogonal channels. The modulation scheme used is Binary Phase Shift Keying (BPSK) for transmission.

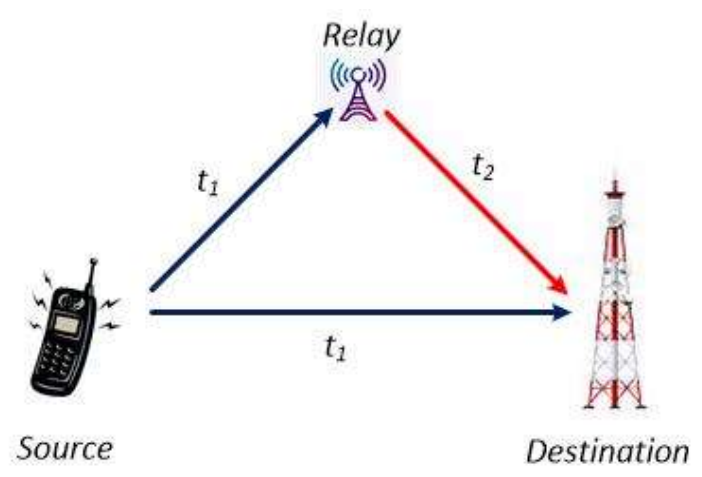

Figure 1. Cooperative Network with single sources, a relay and destination

Let a source signal $m_{i}$ is transmitted from the transmitting source $(S)$. The common equation for the received signal $j_{i}$ is given as:

$$
j_{i}=h_{i} m_{i}+n o_{i}
$$

While, $h_{i} m_{i}$ is the attenuated message signal and denotes the noise.

Relay $\boldsymbol{R}$ is positioned at different locations represented by between source $\boldsymbol{S}$ and destination $\boldsymbol{D}$, whose possible distance from $\mathrm{S}$ and $\mathrm{D}$ can be expressed by:

$$
\begin{aligned}
d_{11} & =\sqrt{\left(d_{1}^{2}+0.25^{2}\right)} \\
d_{12} & =\sqrt{\left(\left(1-d_{1}\right)^{2}+0.25^{2}\right)}
\end{aligned}
$$

$\mathrm{d}_{1}$, shows the point of location $(0.25,0.5,0.75)$ from the source $\mathrm{S}$. The transmission process completes in two time slots (Time Slot I and Time Slot II). In first slot (Time slot I), shown in fig. 2, the source S is communicating with relay $\mathrm{R}$ and destination $\mathrm{D}$ directly.

The total transmitted power $P_{t}$ is taken as 1 . The transmitted power of source is given by:

$$
P_{b}=P_{t} / 2
$$

The transmitted power of relay is given by:

$$
P_{r}=P_{t} / 2
$$


The signals transmitted during time slot I from [14], [15] will be:

Signals received by relay from source:

$$
j_{S R}=\left(d_{k}\right)^{-u} \sqrt{\frac{P_{b}}{2}} \times h \cdot m+\sqrt{n o_{i}} \times n b r
$$

( $i$ = length of SNR which is taken as $40 \mathrm{~dB}$ )

$d_{k}$ shows, the location point, $u$ is the pathloss exponent, $P_{b}$ transmitted power by source, $h . m$ is the attenuated signal, $n o_{i}$ shows the noise power and $n b r$ is the awgn for source relay link.

Signals received by destination from source:

$$
j_{S D}=\sqrt{\frac{P_{b}}{2}} \times h . m+\sqrt{n o_{i}} \times n b m
$$

$\mathrm{nbm}$ is the awgn for source destination link.

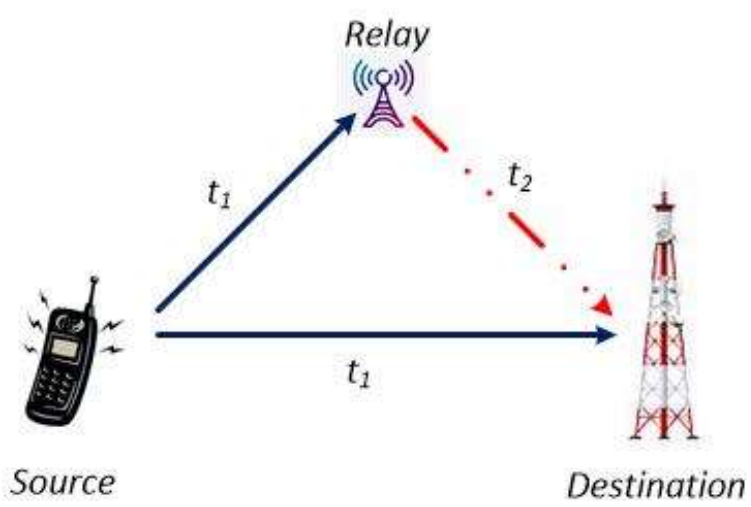

Figure 2. $t_{l}=$ Time Slot I, Source is sending signals to Relay and Destination

While, in the second slot (Time Slot II), drawn in figure 3, relay sends the message signals to destination with the help of AF protocol

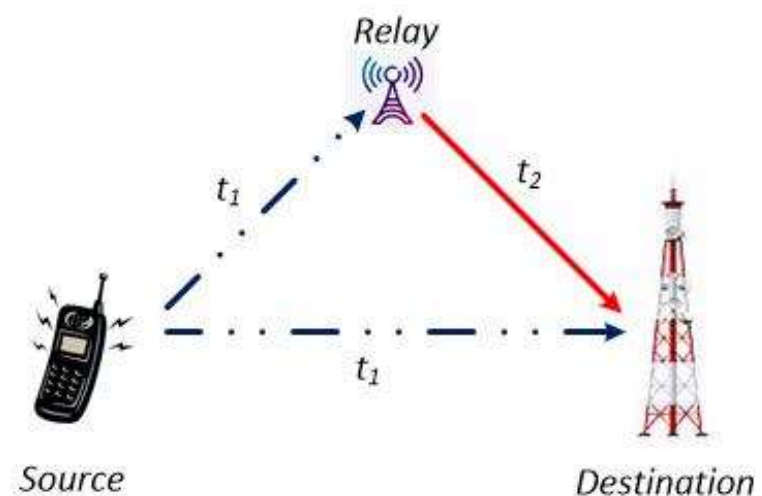

Figure 3. $t_{2}=$ Time Slot II, Relay is sending signals to Destination

2.1. Amplify and Forward (AF). The relay performs amplifications on the signals (originated from the source) and forwards to the desired receiving point. The main disadvantage of AF is in amplification phase, the noise that is present is also amplified. AF is practiced in that condition when relay has limited time for both encoding and decoding. 


$$
\beta_{k}=\sqrt{\frac{\mathrm{P}_{\mathrm{r}}}{\mathrm{A}_{11}^{2} \times \mathrm{P}_{\mathrm{b}}+n o_{i}}}
$$

$\beta_{\mathrm{k}}$ is the amplification factor, $\mathrm{A}_{11}$ is the average channel gain for source relay link. The received data at source from relay station will be:

$$
j_{R D}=\left(d_{k}\right)^{-u} \times \beta_{k} \times h . m+\sqrt{n o_{i}} \times n r m
$$

$\mathrm{nrm}$ is the awgn for relay destination link.

2.2. MRC. The destination combines all the signals by a combining technique called Maximum Ratio Combining (MRC).

$$
M[n]=\sum_{i=1}^{k} j_{S D}[n] \cdot j_{R D}[n]
$$

The normalizing factors $\omega_{1}$ from [14], [15] can be stated as:

$$
\omega_{1}=\sqrt{\frac{E_{R D}}{\left(E_{S R}+n_{o}\right)\left|h \cdot m_{R D}\right|^{2}+1}}
$$

Let, $\omega_{1} \cong \omega$, then the signals received by $\boldsymbol{D}$ from the second slot will be:

$$
C_{2=}\left(\frac{1}{\omega}\left(\left(d_{S R}\right)^{-u}\left(d_{R D}\right)^{-u} \sqrt{\frac{E_{S R} E_{R D}}{E_{S R}+n_{o}}} h \cdot m_{S R} h \cdot m_{R D}\right)\right) a_{1}
$$

Figure 4, displays the locations of $\boldsymbol{R}$ from the $\boldsymbol{S}$ and $\boldsymbol{D}$.

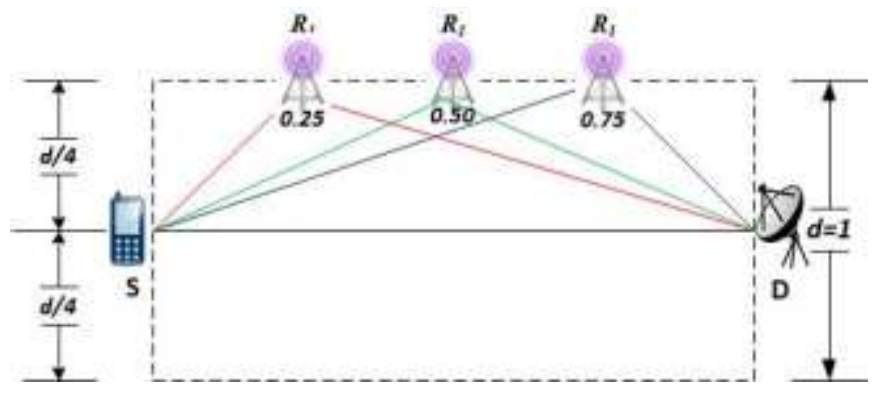

Figure 4. Relays Displacement Optimization

Let $\quad A=\sqrt{E_{S D}} h \cdot m_{S D}$

$$
B=\frac{1}{\omega}\left(\left(\sqrt{\frac{E_{S R} E_{R D}}{E_{S R}+n_{o}}} h \cdot m_{S R} h \cdot m_{R D}\right)\right)
$$

2.3. The Rayleigh Fading Channel: The Rayleigh fading occurs "if there are frequent unintended paths without direct line-of-sight path between source $\boldsymbol{S}$ and destination $\boldsymbol{D}$ ”. The probability density function (PDF) of Rayleigh states the envelope of the generated signals, which is stated as: 


$$
p_{|h[m]|}(y)=\frac{2}{\Omega} \exp \left(-\frac{y^{2}}{\Omega}\right), y \geq 0
$$

Where $\Omega$ represents the average fading power.

2.4. The Rician Fading Channel: This kind of fading develops when "a straight line of sight exists with numerous indirect diverse paths". The Rician PDF explains the envelope of the arriving signals.

$$
p_{|h[m]|}(y)=\frac{2(1+R) y}{\Omega} \exp \left(-R-\frac{(1+R) y^{2}}{\Omega}\right) I_{0}\left(2 y \sqrt{\frac{R(1+R)}{\Omega}}, y \geq 0\right.
$$

Whereas. $I_{0}($.$) is the "zeroth-order"enhanced Bessel function.$

2.5. The Nakagami Fading Channel: This sort of fading evolves as a result of multi-path scattering with comparatively higher delay-time scattering, with several groups of echoed waves. For Nakagami fading model, the PDF can be specified as:

$$
p_{|h[n]|}(x)=\frac{2 m^{m} x^{2 m-1}}{\Omega^{m} \Gamma(m)} \exp \left(-\frac{m x^{2}}{\Omega}\right), x \geq 0
$$

While, the Nakagami fading channel parameter " $\mathrm{m}$ " values from $1 / 2$ to $+\infty$. Moreover, the gamma function $\Gamma($.$) is equal to:$

$$
\Gamma(m)=\int_{0}^{\infty} t^{m-1} \exp (-t) d t=(m-1) !
$$

3. Simulation Outcomes: We will display our simulation effects with the MATLAB software. The system's presentation plots with respect to the average Symbol Error Rate against Signal to Noise Ratio in dB's are drawn. The outcomes are assumedover different fading channels for a BPSK modulation. We assumed that the source (S), destination (D) and a relay (R) have the identical noise variance $N_{o}$. The SNR $\gamma$ can be then calculated as:

$$
\gamma=\alpha^{2} E_{b} / N_{o}
$$

Here, the distance between two source and destination is normalized to 1 for the sake of simplification in the calculations.

Figure 5, shows a general comparison between different channels in our selected scenario but without any distance effect. Comparing the SER values at $10^{-5}$, relay in Nakagami and Rician channels performs far better than Rayleigh fading channel.

Secondly, in figure 6, the average SER performance of relay in Nakagami, Rician and Rayleigh fading channel at a distance of 0.25 from the source is analyzed. Here for SER at $10^{-5}$, we measure SNR values at 10 $\mathrm{dB}, 14.9 \mathrm{~dB}$ and $25 \mathrm{~dB}$ in Rician, Nakagami and Rayleigh fading channels respectively for AF protocol. It is obvious from the simulation results that relay in Rician fading channel performs much better than Nakagami and Rayleigh channels in terms of lower SER values when relay is located at aforementioned distance. 


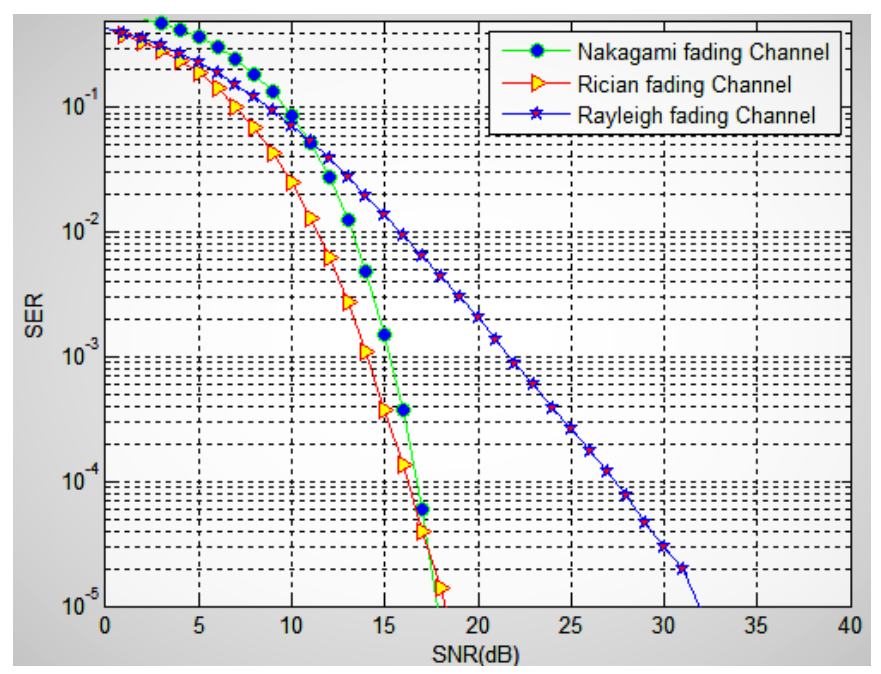

Figure-5: Effect of different channels on the relay assisted transmission

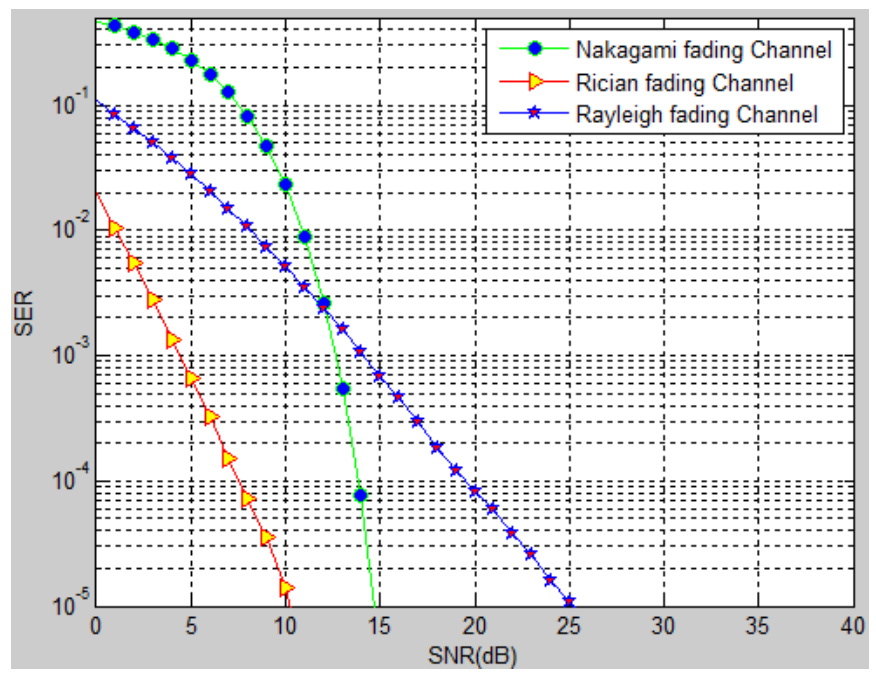

Figure 6. Comparison of relay performance at 0.25 distance from $S$ in different fading channels

Furthermore, in figure 7, we inspect the average SER comparison amongst three selected aforementioned channels when relay position changes to 0.5 from the source. Now keeping SER at $10^{-5}$, the performance of relay at Nakagami fading channel outperforms other channels performance at 0.5 distance. The SNR values for Nakagami, Rician and Rayleigh are approximately $6.5 \mathrm{~dB}, 11.1 \mathrm{~dB}$ and $26 \mathrm{~dB}$ respectively. This evaluation shows that relay in Nakagami fading channel provides promising results at 0.5 distance for the source in AF protocol. 


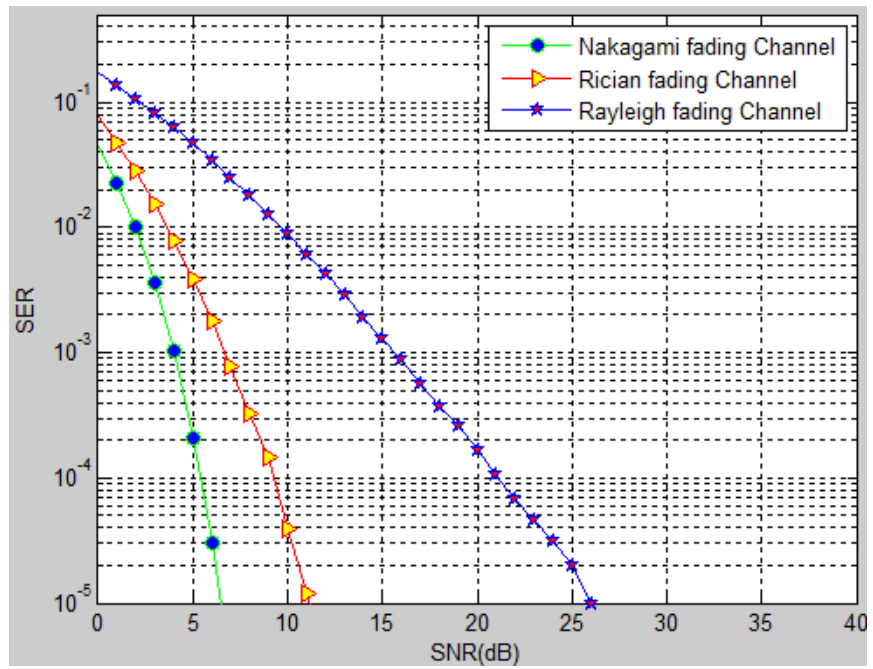

Figure 7. Comparison of relay performance at 0.5 distance from $S$ in different fading channels

Finally, the SER comparison between Nakagami, Rician and Rayleigh channels when relay is at a distance of 0.75 from source and much near to destination is shown in figure 8. It is clearly seen that now for SER value of $10^{-5}$, again Nakagami channel gives better results than the other two channels. Here SNR values for Nakagami, Rician and Rayleigh are $14 \mathrm{~dB}, 16 \mathrm{~dB}$ and $28.9 \mathrm{~dB}$ respectively for $10^{-5}$ SER value.

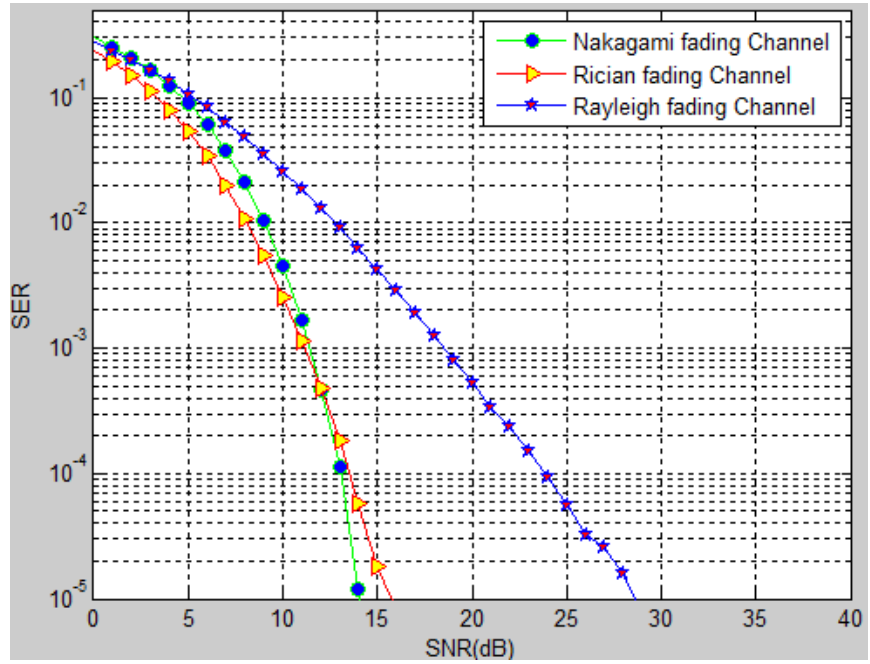

Figure 8. Comparison of relay performance at 0.75 distance from $S$ in different fading channels

4. Discussion: In all our simulation results, it is crystal clear that the performance of relay at any distance from the source is degraded in Rayleigh fading channel in comparison with Nakagami and Rayleigh fading channels which show much improvement in the performance.

5. Conclusion: The Rayleigh, Rician and the Nakagami fading channels for Amplify-and-Forward protocol for various relay location from the source and destination have been considered. MRC approach is used at destination to combine all the signals in order to achieve diversity. The SER vs SNR results are explored for AF protocol in various channels at different location. It is certified that relay location has a main effect on the performance of cooperative transmission at different channels. Additional, in each fading channel relays function better when positioned at the center of communication network. However, 
the performance of cooperative communicationcan be boosted by using different protocols and combining techniques.

\section{REFERENCES}

[1] Nosratinia, A., Hunter, T. E., \& Hedayat, A. (2004). " Cooperative communication in wireless networks". Communications Magazine, IEEE, 42(10), 74-80.

[2] Goldsmith, A. (2005). "Wireless communications. Cambridge university press".

[3] Mogensen, P. E., Koivisto, T., Pedersen, K., Kovács, I. Z., Raaf, B., Pajukoski, K., \& Rinne, M. J. (2009, May). "LTE-advanced: the path towards gigabit/s in wireless mobile communications". In Wireless Communication, Vehicular Technology, Information Theory and Aerospace\& Electronic Systems Tech, 2009. Wireless VITAE 2009. 1st International Conference on (pp. 147-151). IEEE.

[4] Zhao, Y., Adve, R., \& Lim, T. J. (2006). "Symbol error rate of selection amplify-and-forward relay systems". Communications Letters, IEEE, 10(11), 757-759.

[5] Atapattu, S., \& Rajatheva, N. (2007, October). "Exact SER of Alamouti code transmission through amplify-forward cooperative relay over Nakagami-m fading channels". In Communications and Information Technologies, 2007. ISCIT'07. International Symposium on (pp. 1429-1433). IEEE.

[6] Louie, R. H., Li, Y., \& Vucetic, B. (2008, May). "Performance analysis of beamforming in two hop amplify and forward relay networks. In Communications", 2008. ICC'08. IEEE International Conference on (pp. 4311-4315). IEEE.

[7] Wu, Y., \& Pätzold, M. (2008, November). "Performance analysis of amplify-and-forward cooperative communication systems with channel estimation errors". In Communication Systems, 2008. ICCS 2008. 11th IEEE Singapore International Conference on (pp. 1620-1624). IEEE.

[8] Maham, B., \& Hjørungnes, A. (2009). "Performance analysis of amplify-and-forward opportunistic relaying in Rician fading”. Signal Processing Letters, IEEE, 16(8), 643-646.

[9] Maham, B., \& Hjørungnes, A. (2009). "Asymptotic performance analysis of amplify-and-forward cooperative networks in a Nakagami-m fading environment". Communications Letters, IEEE, 13(5), 300-302.

[10] Song, L. (2011). "Relay selection for two-way relaying with amplify-and-forward protocols". Vehicular Technology, IEEE Transactions on, 60(4), 1954-1959.

[11] Katti, S., Gollakota, S., \& Katabi, D. (2007, August). "Embracing wireless interference: Analog network coding”. In ACM SIGCOMM Computer Comm Review (Vol. 37, No. 4, pp. 397-408). ACM.

[12] Zhang, S., Liew, S. C., \& Lam, P. P. (2006, September). Hot topic: physical-layer network coding. In Proceedings of the 12th annual international conference on Mobile computing and networking (pp. 358-365). ACM.

[13] Salman, S., Rahman, A., \& Khan, I. (2015). Comparison of Cooperative Diversity Protocols in Various Relay Locations through Network Coding. VFAST Transactions on Software Engineering, 8(1), 01-09.

[14] Nabar, R. U., Bölcskei, H., \& Kneubühler, F. W. (2004). "Fading relay channels: performance limits and space-time signal design”. Selected Areas in Communications, IEEE Journal on, 22(6), 1099-1109.

[15] Atapattu, S., Rajatheva, N., \& Tellambura, C. (2010). "Performance Analysis of TDMA Relay Protocols Over Nakagami-Fading". Vehicular Technology, IEEE Transactions on, 59(1), 93-104. 